indeed to promote sensitivity to antiandrogen therapy at late stages.

\section{Nuclear hormone receptor regulation by RB/E2F in other cancers}

Finally, if RB modulates the expression and activity of AR in prostate cancer, are other nuclear hormone receptors regulated by $\mathrm{RB} / \mathrm{E} 2 \mathrm{Fs}$ in other cancers, and does the efficacy of relevant endocrine therapies depend upon RB status? Early loss of RB is generally linked to responsiveness to conventional genotoxic therapies in various tumor types, but is the opposite true for endocrine therapy? For example, does RB loss mitigate against hormone therapy in breast cancer, as has been suggested previously (14)? Previous work has also identified a direct interaction between RB and the glucocorticoid receptor (15). Does this come into play in modulating stress responses in cancer etiology? Overall, the work of Sharma and colleagues (4) raises many intriguing questions that have major implications for how we diagnose and treat prostate cancer, but to what extent we must reconsider a role for
$\mathrm{RB}$ in the late stages of and for therapy of other human cancers remains to be seen.

\section{Acknowledgments}

The author's work is supported by NIH grant NCI-RO1-CA131188.

Address correspondence to: Kay F. Macleod, Ben May Department for Cancer Research, Gordon Center for Integrative Sciences, W-338, University of Chicago, 929 E. 57th Street, Chicago, Illinois 60637, USA. Phone: 773.834.8309; Fax: 773.702.4476; E-mail: kmacleod@uchicago.edu.

1. Burkhart DL, Sage J. Cellular mechanisms of tumour suppression by the retinoblastoma gene. Nat Rev Cancer. 2008;8(9):671-682.

2. Chen HZ, Tsai SY, Leone G. Emerging roles of E2Fs in cancer: an exit from cell cycle control. Nat Rev Cancer. 2009;9(11):785-797.

3. Okamoto A, et al. Mutations and altered expression of p16INK4a in human cancer. Proc Natl Acad Sci US A. 1994;91(23):11045-11049.

4. Sharma A, et al. The retinoblastoma tumor suppressor controls androgen signaling and human prostate cancer progression. J Clin Invest. 2010; 120(12):4478-4492.

5 . Huggins $\mathrm{CB}$, Hodges CV. Studies on prostatic cancer I: The effect of castration, of estrogen and of andro- gen injection on serum phsophatase in metastatic carcinoma of prostate. Cancer Res. 1941;1:293-297.

6. Shen MM, Abate-Shen C. Molecular genetics of prostate cancer: new prospects for old challenges. Genes Dev. 2010;24(18):1967-2000.

7. Taylor BS, et al. Integrative genomic profiling of human prostate cancer. Cancer Cell. 2010;18(1):11-22.

8. Wang QJ, et al. Androgen recpetor regulates a distinct transcription program in androgen-independent prostate cancer. Cell. 2009;138(2):245-256.

9. Foster CS, et al. Transcription factor E2F3 overexpressed in prostate cancer independently predicts clinical outcome. Oncogene. 2004;23(35):5871-5879.

10. Lee EY, Cam H, Ziebold U, Rayman JB, Lees JA, Dynlacht BD. E2F4 loss suppresses tumorigenesis in Rb mutant mice. Cancer Cell. 2002;2(6):463-472.

11. Ianari A, et al. Proapoptotic function of the retinoblastoma tumor suppressor protein. Cancer Cell. 2009;15(3):184-194.

12. Tran C, et al. Development of a second-generation antiandrogen for treatment of advanced prostate cancer. Science. 2009;324(5928):787-790.

13. Knudson AG. Mutation and cancer: statistical study of retinoblastoma. Proc Natl Acad Sci U S A. 1971;68(4):820-823.

14. Bosco EE, et al. The retinoblastoma tumor suppressor modifies the therapeutic response of breast cancer. J Clin Invest. 2007;117(1):218-228.

15. Singh P, Coe J, Hong W. A role for retinoblastoma protein in potentiating transcriptional activation by the glucocorticoid receptor. Nature. 1995 ; 374(6522):562-565

16. Weinberg RA. The retinoblastoma protein and cell cycle control. Cell. 1995;81(3):323-330.

\title{
Which species are in your feces?
}

\section{Colby Zaph}

\author{
Biomedical Research Centre, Department of Pathology and Laboratory Medicine, University of British Columbia, Vancouver, British Columbia, Canada.
}

\begin{abstract}
Nosocomial infections (i.e., infections acquired as a result of treatment in a hospital or health care unit) result in approximately 100,000 deaths and cost more than 25 billion dollars per year in the US alone. These infections are caused primarily by bacteria and affect mainly immunosuppressed patients. However, not all patients acquire infections, and the events leading up to infection are unclear. In this issue of the JCI, Ubeda et al. report how acquisition of one such infection, vancomycin-resistant Enterococcus faecium (VRE), is linked to a shift in the microbial flora following antibiotic treatment. This study highlights the potential for high-throughput sequencing of intestinal microbiota as a means to identify high-risk populations.
\end{abstract}

Nosocomial infections are infections acquired as a result of treatment in a hospital or health care unit. They are increasingly becoming a significant public health problem. It is thought that $5 \%-10 \%$ of patients entering hospitals worldwide develop a hospital-acquired infection (HAI) (1). The global growth of antibiotic-resistant bacterial strains, such as methicillin-resistant

Conflict of interest: The author has declared that no conflict of interest exists.

Citation for this article: J Clin Invest. doi:10.1172/ JCI45263.
Staphylococcus aureus (MRSA) and vancomycin-resistant Enterococcus faecium (VRE), has complicated the control of HAIs and highlights the need for a better understanding of how HAIs develop, are acquired, and may be controlled.

Intestinal Enterococcus infections are among the most common type of infection acquired by hospitalized patients. While enterococcal infections are much less capable of causing disease than HAIs such as Clostridium difficile and S. aureus, they still cause complications, prolong hospital stays, and can be fatal, particularly those caused by VRE. Fatal VRE infections, which occur upon bacterial invasion of the bloodstream, primarily affect immunocompromised patients such as those undergoing bone marrow or hematopoietic stem cell transplantation (HSCT) (2). It has been estimated that up to $50 \%$ of HSCT patients acquire VRE, and infection with VRE is independently associated with increased mortality in these patients (3). Identifying high-risk patients and developing novel methods to combat VRE are critical components in combating HAIs. The study by Ubeda et al. in this issue of the JCI begins to address this in both mice and humans using high-throughput sequencing analysis of commensal flora populations (4).

\section{Commensal flora and susceptibility to infection}

Over the past several years, interest in understanding the relationship between the commensal flora and disease states has increased markedly. It is clear that changes in the flora have a dramatic effect on the 
A Unperturbed

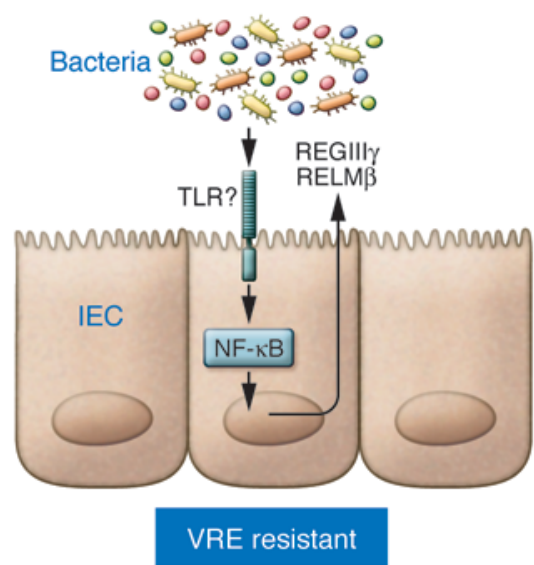

B Antibiotic treatment

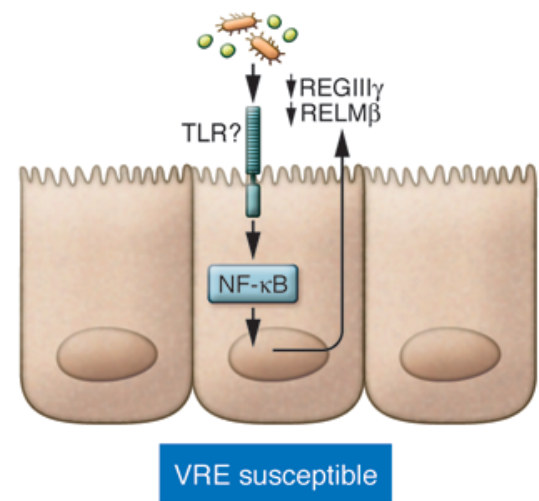

C Recovery

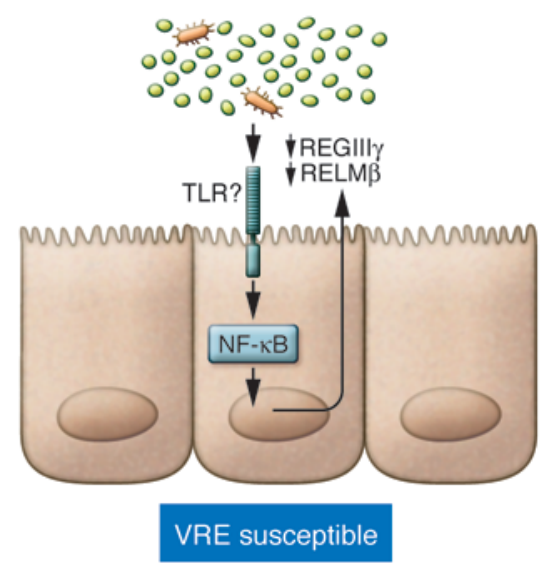

\section{Figure 1}

Antibiotic treatment leads to long-term changes in the commensal flora and susceptibility to VRE. (A) In the steady state, interactions between commensal bacteria and host cells in the intestine - primarily epithelial cells - result in the production of antimicrobial effector proteins (AMPs), such as REGIIl $\gamma$ and RELM $\beta$, that shape the commensal populations. The production of AMPs in this context may be dependent on $\mathrm{NF}-\mathrm{KB}$, which is presumed to be activated by TLR recognition of commensal bacterial products. In this state, mice are resistant to VRE infection. (B and C) Following transient antibiotic treatment, the numbers and diversity of the bacterial populations are severely reduced. Antibiotic treatment - presumably through a reduction in commensal bacteria - also results in decreased expression of AMPs. These changes result in long-lived increased susceptibility to VRE.

outcomes of many diseases and directly or indirectly affect immune homeostasis and function (5). For example, mice lacking all commensal bacteria (germ-free mice) have dramatically altered immune systems (6, $7)$ and immune responses at enteric and peripheral sites $(8,9)$. Furthermore, it has been shown in humans and animals that antibiotic-induced changes in the commensal flora are dramatic and long-lived (10) and have consequences for susceptibility to intestinal infections, including VRE (11).

While the exact role of the commensal flora in intestinal homeostasis is unclear, there are several studies that have identified components of this regulatory network (Figure 1). First, interactions between the host and the commensal flora via MyD88-dependent Toll-like receptor (TLR) activation are critical for maintenance of intestinal homeostasis $(12,13)$. Whether commensal bacteria are recognized by intestinal epithelial cells (IECs) or by intestinal antigen-presenting cells such as DCs and macrophages is not clear. However, mice with an IEC-specific deletion of the gene encoding IкB kinase- $\gamma$ (IKK- $\gamma$; also known as NEMO) spontaneously develop intestinal inflammation (14), suggesting that NF-кB activation - possibly initiated by TLR activation by commensal bacteria - is playing a critical role in maintaining the host-commensal homeostatic relationship. The regulatory role of com- mensal bacteria may be to activate the intestinal immune response to control potentially pathogenic bacteria, as it has been shown that colonization of germ-free mice with a defined flora leads to the expression of several antimicrobial peptides, including the secreted C-type lectin regenerating islet-derived III $\gamma$ (REGIII $\gamma$ ), which is directly toxic to Gram-positive commensal and pathogenic bacteria $(15,16)$, and the goblet cell-specific protein resistin-like molecule- $\beta$ (RELM $\beta$ ) (17). In addition, antibiotic treatment of mice leads to a reduction in total numbers of bacteria, loss of REGIII $\gamma$ and RELM $\beta$ expression $(11,18)$, and increased susceptibility to outgrowth of, or infection with, intestinal bacterial pathogens such as Salmonella typhimurium (19-21) and VRE (11). Thus, the commensal flora are a critical component in maintaining the steady state as well as in preventing the outgrowth of potentially pathogenic species. However, the dynamics of the commensal flora prior to colonization by a pathogenic species is not known, and whether any predictions about disease progression can be made is unclear.

\section{Antibiotics drastically change the microbiome}

High-throughput pyrosequencing of bacterial 16S rDNA sequences allows for nonbiased, global analysis of the relative composition of bacterial populations in vivo. Using this technique, Ubeda et al. characterized the effects of several antibiotic regimens on the composition of the intestinal microbiome in mice (4); that is, they investigated how antibiotic treatment affected the relative frequencies of bacterial populations by quantifying the genomes of all the microorganisms that live in the intestine of a healthy adult mouse (the microbiome). Interestingly, a transient 7-day antibiotic treatment resulted in a dramatic shift in the commensal populations, with the outgrowth of several minor populations of bacteria, including Enterobacteriaceae, Clostridium, and Enterococcus species, bacteria that tend to be causative agents of nosocomial infections. Furthermore, several populations (primarily from the Bacteroidetes phylum) failed to recover after antibiotic withdrawal. These changes in the flora allowed normally resistant mice to become susceptible to VRE infection. Thus, the composition of the bacterial flora was critical in restraining the outgrowth of potentially pathogenic species, a pattern that has also been observed in animals with S. typhimurium infection (19-21).

Consistent with previous studies (10), these changes in bacterial populations observed by Ubeda and colleagues were long-lived and failed to return to normal, even after several months (4). One striking finding in the study of Ubeda et al. was 
A Healthy

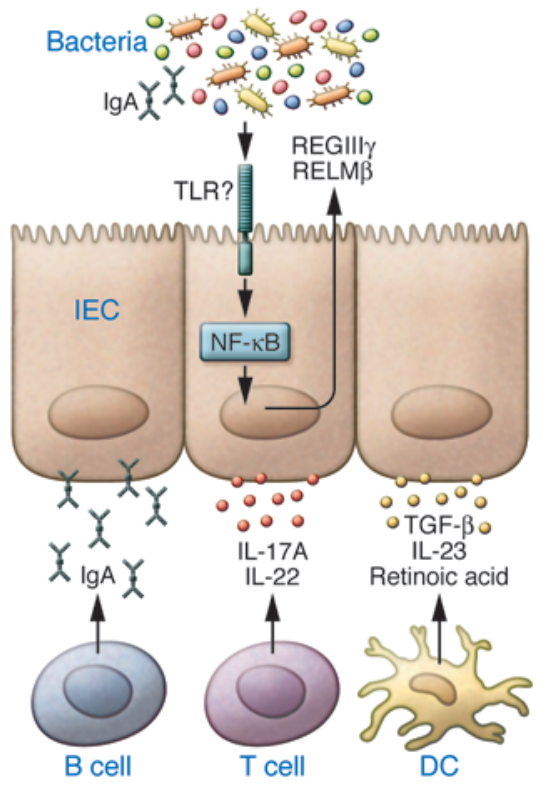

that, even 2 months after antibiotic treatment, mice were still susceptible to VRE infection. These results suggest that transient antibiotic treatments result in longterm changes in the commensal bacterial populations that predispose the host to certain infections. Although the molecular mechanisms associated with this increased susceptibility remain unclear, the potential identification of a signature at the level of bacterial populations may provide a novel analytical tool to identify patients predisposed to fatal VRE infections.

\section{Relevance to human disease}

To directly test whether a bacterial population signature could identify patients predisposed to fatal VRE infections, Ubeda et al. examined the microbiomes of human patients undergoing allogeneic HSCTs, a group at high risk for developing HAIs (4). All HSCT patients received prophylactic antibiotic treatment upon admission, during fever episodes, and at any point when neutrophil numbers decreased, so as to protect them against potential bacterial infections caused by neutropenia. Antibiotic use was not consistent, although most patients received ciprofloxacin, vancomycin, and trimethoprim-sulfamethoxazole at some point during the observation period. During the course of the study, two patients developed systemic VRE infections. The patients that developed VRE bacteremia displayed a striking change in
B Immunosuppressed

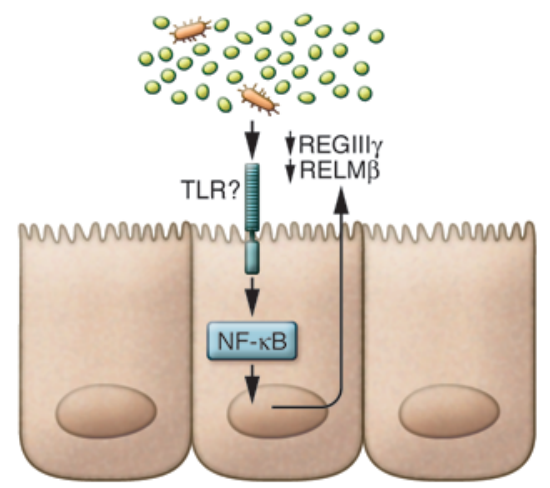

\section{Figure 2}

The role of the immune system in regulation of the commensal microflora. (A) In healthy subjects, recovery from antibiotic treatment results in minor changes to the diversity of the commensal flora. Immune mechanisms, including production of commensal-specific $\lg \mathrm{A}$, effector and regulatory cytokines, and retinoic acid that can act on IECs and other immune cells, may all contribute to the maintenance of intestinal homeostasis. (B) In contrast, immunocompromised patients that have impaired B cell, T cell, and DC responses often do not recover bacterial diversity following antibiotic treatment; in some cases, a single genus dominates the flora. the intestinal flora immediately prior to VRE infection, with a total shift toward bacteria from the genus Enterococcus as the dominant microbial population. Thus, the intestinal domination by Enterococcus species that preceded bloodstream invasion suggests that monitoring of fecal bacterial populations may provide a warning of impending systemic infections in immunocompromised patients and offer an opportunity for therapeutic intervention.

The shift in commensal flora to a single genus in immunocompromised patients is in striking contrast to data in healthy volunteers treated with ciprofloxacin, who lost bacterial diversity but did not acquire overgrowth by a single species (10). This highlights the important role of the host immune response in regulating commensal bacterial populations (Figure 2). The production of immune mediators in the gut - such as IgA (22); effector T cell- and DC-derived cytokines, including IL-17A, IL-22, and IL-23 $(23,24)$; and regulatory molecules, including TGF $\beta$ and retinoic acid (25) - may all contribute to maintaining a balanced commensal flora. Thus, the status of the immune system is also a critical component in commensal homeostasis, further highlighting the closely linked host-commensal relationship.

\section{Future studies}

The study by Ubeda and colleagues (4) raises questions about the current use of antibiotics and how at-risk patients are identified. Antibiotic treatment of highrisk patients will not stop, but a closer examination of the effects of different classes of antibiotics on commensal populations and their subsequent consequences on infections should be investigated. While Ubeda et al. focused on VRE (4), other HAIs may not behave similarly, and thus global strategies may not apply. However, the use of high-throughput sequencing allows for a nonbiased global analysis of commensal populations, thereby potentially identifying distinct signatures in other HAIs. Furthermore, it is clear that the interactions between the commensal flora and the host immune system are bidirectional, with important ramifications for intestinal homeostasis. Future studies correlating intestinal and peripheral immune responses with commensal signatures may provide novel methods not only to affect the commensal flora, but also to regulate immune responses through manipulation of commensal populations.

\section{Acknowledgments}

The author is funded by Canadian Institutes of Health Research grants MSH95368, MOP-89773, and MOP-106623 and by Canada Foundation for Innovation.

Address correspondence to: Colby Zaph, Biomedical Research Centre, Department of Pathology and Laboratory Medicine, 
University of British Columbia, Vancouver, British Columbia V6T 1Z3, Canada. Phone: 604.822.7231; Fax: 604.822.7815; E-mail: colby@brc.ubc.ca.

1. Scott RD. The Direct Medical Costs of HealthcareAssociated Infections in U.S. Hospitals and the Benefits of Prevention, 2009. Division of Healthcare Quality Promotion, National Center for Preparedness, Detection, and Control of Infectious Diseases, Coordinating Center for Infectious Diseases, Centers for Disease Control and Prevention, February 2009.

2. Magauran CE, Salgado CD. Challenges and advances in infection control of hematopoietic stem cell transplant recipients [published online ahead of print August 10, 2010]. Infect Disord Drug Targets. doi:BSP/ID DT /E-Pub/-0039-10-5 [pii].

3. Salgado CD, Ison MG. Should clinicians worry about vancomycin-resistant Enterococcus bloodstream infections? Bone Marrow Transplant. 2006; 38(12):771-774.

4. Ubeda C, et al. Vancomycin-resistant Enterococcus domination of intestinal microbiota is enabled by antibiotic treatment in mice and precedes bloodstream invasion in humans. J Clin Invest. 2010; 120(12):4332-4341.

5. Artis D. Epithelial-cell recognition of commensal bacteria and maintenance of immune homeostasis in the gut. Nat Rev Immunol. 2008;8(6):411-420.

6. Zaph C, et al. Epithelial-cell-intrinsic IKK-beta expression regulates intestinal immune homeostasis. Nature. 2007;446(7135):552-556.

7. Ivanov II, et al. Induction of intestinal Th17 cells by segmented filamentous bacteria. Cell. 2009; 139(3):485-498.
8. Lee YK, Menezes JS, Umesaki Y, Mazmanian SK. Microbes and Health Sackler Colloquium: Proinflammatory $\mathrm{T}$-cell responses to gut microbiota promote experimental autoimmune encephalomyelitis [published online ahead of print July 28, 2010]. Proc Natl Acad Sci U S A. doi:10.1073/ pnas.1000082107.

9. Stepankova R, et al. Segmented filamentous bacteria in a defined bacterial cocktail induce intestinal inflammation in SCID mice reconstituted with CD45RBhigh CD4+ T cells. Inflamm Bowel Dis. 2007; 13(10):1202-1211.

10. Dethlefsen L, Huse S, Sogin ML, Relman DA. The pervasive effects of an antibiotic on the human gut microbiota, as revealed by deep $16 \mathrm{~S}$ rRNA sequencing. PLoS Biol. 2008;6(11):e280.

11. Brandl K, et al. Vancomycin-resistant enterococci exploit antibiotic-induced innate immune deficits. Nature. 2008;455(7214):804-807.

12. Rakoff-Nahoum S, Paglino J, Eslami-Varzaneh F, Edberg S, Medzhitov R. Recognition of commensal microflora by toll-like receptors is required for intestinal homeostasis. Cell. 2004;118(2):229-241.

13. Rakoff-Nahoum S, Hao L, Medzhitov R. Role of toll-like receptors in spontaneous commensaldependent colitis. Immunity. 2006;25(2):319-329.

14. Nenci A, et al. Epithelial NEMO links innate immunity to chronic intestinal inflammation. Nature. 2007;446(7135):557-561.

15. Keilbaugh SA, et al. Activation of RegIIIbeta/gamma and interferon gamma expression in the intestinal tract of SCID mice: an innate response to bacterial colonisation of the gut. Gut. 2005;54(5):623-629.

16. Cash HL, Whitham CV, Behrendt CL, Hooper LV. Symbiotic bacteria direct expression of an intestinal bactericidal lectin. Science. 2006;
313(5790):1126-1130

17. Wang ML, et al. Regulation of RELM/FIZZ isoform expression by $\mathrm{Cdx} 2$ in response to innate and adaptive immune stimulation in the intestine. Am J Physiol Gastrointest Liver Physiol. 2005;288(5):G1074-G1083.

18. Hill DA, et al. Metagenomic analyses reveal antibioticinduced temporal and spatial changes in intestinal microbiota with associated alterations in immune cell homeostasis. Mucosal Immunol. 2010;3(2):148-158.

19. Sekirov I, et al. Antibiotic-induced perturbations of the intestinal microbiota alter host susceptibility to enteric infection. Infect Immun. 2008; 76(10):4726-4736.

20. Croswell A, Amir E, Teggatz P, Barman M, Salzman $\mathrm{NH}$. Prolonged impact of antibiotics on intestinal microbial ecology and susceptibility to enteric Salmonella infection. Infect Immun. 2009;77(7):2741-2753.

21. Garner CD, et al. Perturbation of the small intestine microbial ecology by streptomycin alters pathology in a Salmonella enterica serovar typhimurium murine model of infection. Infect Immun. 2009; 77(7):2691-2702.

22. Cerutti A, Rescigno M. The biology of intestinal immunoglobulin A responses. Immunity. 2008; 28(6):740-750.

23. Strober W. The multifaceted influence of the mucosal microflora on mucosal dendritic cell responses. Immunity. 2009;31(3):377-388.

24. Abt MC, Artis D. The intestinal microbiota in health and disease: the influence of microbial products on immune cell homeostasis. Curr Opin Gastroenterol. 2009;25(6):496-502.

25. Mucida D, Park Y, Cheroutre H. From the diet to the nucleus: vitamin A and TGF-beta join efforts at the mucosal interface of the intestine. Semin Immunol. 2009;21(1):14-21.

\section{Integral role of integrins in Th17 development}

\section{Derek A. Pociask and Jay K. Kolls}

Department of Genetics, Louisiana State University, Health Sciences Center, New Orleans, Louisiana, USA.

\begin{abstract}
A lineage of $\mathrm{CD}^{+} \mathrm{T}$ cells known as Th17 cells, which are derived by exposure of naive $\mathrm{CD}^{+} \mathrm{T}$ cells to IL- 6 and TGF- $\beta$, have been implicated in several autoimmune diseases. In this issue of the JCI, studies by Acharya et al. and Melton et al. show that TGF- $\beta$ is activated at the DC/CD4 ${ }^{+} \mathrm{T}$ cell synapse by $\alpha v$ integrins and that this activation is required for Th17 differentiation and autoimmunity in the central nervous system. Thus, these studies offer a potential therapeutic target in fighting autoimmune diseases.
\end{abstract}

Th17 cells are a recently identified and critical component of the adaptive immune system (1-3). They are characterized by the production of IL-17A and IL-17F as well as other cytokines such as IL-22. These effector cytokines have been shown to be critical for clearance of certain bacteria and fungal pathogens (4). In addition, vaccine-induced Th17 cells have been shown to have broad protective roles against extracellular patho-

Conflict of interest: The authors have declared that no conflict of interest exists.

Citation for this article: J Clin Invest. doi:10.1172/ JCI45450. gens such as Streptococcus pneumoniae and to control Th1 cell migration in the context of vaccination against the intracellular pathogen Mycobacterium tuberculosis (4). However, this protective aspect of the Th17 lineage comes at a cost, as these cells have been implicated in autoimmune diseases such as multiple sclerosis, psoriasis, and rheumatoid arthritis (1-3).

Several groups have shown that naive $\mathrm{CD}^{+} \mathrm{T}$ cells differentiate into Tregs in the presence of TGF- $\beta(5,6)$. However, in the presence of TGF- $\beta$ and IL- 6 , naive CD $4^{+}$ $\mathrm{T}$ cells differentiate into Th17 cells (6-8). Early work by Li et al. (9) showed that the source of TGF- $\beta$ in this context was the $\mathrm{CD}^{+} \mathrm{T}$ cell. However, TGF- $\beta$ is secreted from cells in an inactive form, in which bioactive TGF- $\beta$ is in a complex with its latencyassociated peptide (LAP) through noncovalent bonds. Two studies in this issue of the JCI demonstrate that DCs activate TGF- $\beta$ in an integrin-dependent fashion $(10,11)$, suggesting that the activation of TGF- $\beta$ occurs at the DC/T cell synapse (Figure 1 ) and that this activation is required to drive the differentiation of Th17 T cells.

\section{TGF- $\beta$ and integrins}

TGF- $\beta$ is a multifunctional cytokine involved in many aspects of immunology, angiogenesis, and epithelial growth as well as in pathogenic states such as fibrosis (12). Activation of TGF- $\beta$ has been an area of intense study. Mechanisms identified as leading to the disruption of the noncovalent interaction between LAP and bioactive TGF- $\beta$ and thus activation of TGF- $\beta$ 